\section{Uma análise das operadoras de planos próprios de saúde dos hospitais filantrópicos no Brasil}

\author{
An analysis of managed care provided by \\ charitable hospitals in Brazil
}

\author{
${ }_{1}$ Escola Nacional de Saúde \\ Pública Sergio Arouca, \\ Fundação Oswaldo Cruz, \\ Rio de Janeiro, Brasil. \\ Correspondência \\ M. A. D. Ugá \\ Departamento de \\ Administração e \\ Planejamento em Saúde, \\ Escola Nacional de Saúde \\ Pública Sergio Arouca, \\ Fundação Oswaldo Cruz. \\ Rua Leopoldo Bulhões 1480, \\ Rio de Janeiro, $R J$ \\ 21041-210, Brasil. \\ domingue@ensp.fiocruz.br
}

\begin{abstract}
This article describes charitable hospitals in Brazil that provide managed care and the health management organizations themselves, considering the level of autonomy by the latter in relation to the hospitals and their degree of management development, based on a nationwide study. A random sample of individual hospitals was drawn from the hospital groups. After refusals and replacements, the final sample consisted of 112 individual hospitals and 10 hospital groups. The charitable hospitals' managed care plans do no operate precisely according to the overall Brazilian health plan market, in which most of the managed care is situated in insurance companies, group medicine, and medical cooperatives. Rather than operating as typical plans, they function "inside the organization or hospital itself", almost always with a limited management infrastructure and with little autonomy in relation to the organizations harboring them. Individual plans were more common than collective products, unlike the rest of the market, which may also result from the limited management capacity of these arrangements.
\end{abstract}

Health Plans; Voluntary Hospitals; Hospital Care

\author{
Maria Alicia D. Ugá 1 \\ Sheyla Maria Lemos Lima ${ }^{1}$ \\ Margareth Crisóstomo Portela 1 \\ Miguel Murat Vasconcellos 1 \\ Pedro Ribeiro Barbosa 1 \\ Silvia Gerschman ${ }^{1}$
}

\section{Introdução}

O setor filantrópico hospitalar constitui-se em um segmento pequeno no mercado de operadoras de planos de saúde no Brasil, cobrindo cerca de 3,7\% dos beneficiários da saúde suplementar 1 . No entanto, apreender as características dessa atividade no âmbito desses hospitais ganha relevância pela importância estratégica do setor filantrópico hospitalar para as políticas públicas de saúde. O setor agrega um terço dos leitos hospitalares, é amplamente capilarizado no país e, em muitos municípios, corresponde ao único hospital existente. Além disso, sua tripla condição de prestador público de serviços, através do Sistema Único de Saúde (SUS), e de prestador e operador de planos de saúde, confere-lhe uma condição diferenciada na perspectiva da formulação e implementação de políticas de saúde, considerando o SUS e a saúde suplementar 2 .

O surgimento dos planos de saúde vinculados aos hospitais filantrópicos antecede a conformação do setor de saúde suplementar, tendo em vista que as filantrópicas operavam historicamente com "planos de associados", especialmente entre os hospitais beneficentes, com foco em comunidades específicas tais como religiosas, grupos de imigrantes e outras. Entretanto, ocorreu nos últimos vinte anos um movimento por parte de hospitais filantrópicos que, motivados pela busca de fontes adicionais de recursos, pas- 
saram a comercializar planos próprios de saúde. Hoje coexistem, nesse segmento hospitalar, estruturas de oferta de planos de saúde bastante diferenciadas.

A operação de planos de saúde no país, apesar de realizada há quase quarenta anos, só começou a ser regulada em 1998, a partir da promulgação da Lei $n^{o} .9 .656$ 3, que disciplinou o funcionamento dos planos de saúde e a atuação das empresas que poderiam ser constituídas como suas operadoras. A lei definiu operadoras de planos e seguros privados de saúde como toda e qualquer pessoa jurídica de direito privado, independente da forma jurídica de sua constituição, que ofereça planos ou seguros de saúde, mediante, no primeiro caso, contraprestações pecuniárias com atendimento em serviços próprios ou de terceiros, e, no segundo caso, livre escolha pelo segurado do prestador do respectivo serviço e reembolso de despesas. Em 2001, a Medida Provisória $n^{o}$. 2.177-44 imprimiu mais especificidade à lei, delimitando a operadora de plano de assistência à saúde como pessoa jurídica constituída sob a modalidade de sociedade civil ou comercial, cooperativa, ou entidade de autogestão, que opere produto, serviço ou contrato relativo à prestação continuada de serviços ou cobertura de custos assistenciais a preço pré ou pós-estabelecido, por prazo indeterminado 4 . A medida provisória ainda definiu que o produto ofertado pela operadora, o plano privado de assistência à saúde, deve "garantir, sem limite financeiro, a assistência à saúde, pela faculdade de acesso e atendimento por profissionais ou serviços de saúde, livremente escolhidos, integrantes ou não de rede credenciada, contratada ou referenciada, visando à assistência médica, hospitalar e odontológica, a ser paga integral ou parcialmente às expensas da operadora contratada, mediante reembolso ou pagamento direto ao prestador, por conta e ordem do consumidor".

Em 2000, foi criada a Agência Nacional de Saúde Suplementar (ANS), autarquia vinculada ao Ministério da Saúde, como "órgão de regulação, normatização, controle e fiscalização da assistência suplementar à saúde" 5. A regulamentação gerou um considerável reordenamento na saúde suplementar e, para se registrar na ANS, as operadoras passaram a ter de cumprir um conjunto de requisitos para o qual muitas não estavam adequadamente estruturadas. Têm sido instituídas exigências crescentes de prestação de contas, impondo às operadoras a necessidade de profissionalizar sua gestão para responder às demandas da ANS, seja no campo econômico-financeiro, seja no âmbito da assistência ofertada.

Este artigo objetiva caracterizar os hospitais filantrópicos com operadoras de planos de saúde, bem como as operadoras em si, considerando o nível de autonomia em relação às entidades hospitalares nas quais se inserem e o grau de desenvolvimento gerencial que apresentam. Com base em tal caracterização, também pretende contribuir com reflexões acerca do quanto as operadoras filantrópicas e os produtos por elas ofertados constituem-se, de fato, em operadoras e produtos tal como definidos pela regulamentação e acerca das peculiaridades deste segmento no mercado de saúde suplementar.

\section{Métodos}

A pesquisa foi desenvolvida por meio de um inquérito de abrangência nacional, caracterizandose como um estudo transversal.

O universo do estudo constituiu-se de 163 operadoras de hospitais filantrópicos prestadores de serviços ao SUS, entre as quais 150 vinculadas a hospitais individuais e 13 a conglomerados hospitalares - entidades constituídas por pelo menos dois hospitais. O propósito inicial foi o de construir uma amostra de 130 unidades, por exigência contratual, incluindo todos os conglomerados e uma amostra aleatória de 117 hospitais individuais, estratificados segundo localização geográfica (região metropolitana $v s$. fora da região metropolitana) e, considerando casos em que planos são comercializados sem que a entidade filantrópica tenha necessariamente se constituído como uma operadora e obtido registro na ANS, segundo condição de registro da operadora junto à ANS (registrada $v s$. não registrada).

No contato com as unidades selecionadas, três conglomerados e 38 hospitais individuais se recusaram a participar, os quais foram substituídos, acabando por esgotar o universo de base. Restaram então, dez conglomerados e 112 hospitais individuais que aceitaram participar.

Os dados foram coletados por pesquisadores de campo, especificamente treinados para esta atividade, por meio de entrevista, previamente agendada, com os dirigentes da operadora e do hospital. Foi utilizado um questionário semi-estruturado com questões relacionadas à entidade filantrópica, ao hospital, à operadora e aos seus produtos (planos comercializados).

Em relação à entidade foram investigadas as seguintes condições: aspectos institucionais e econômico-financeiros das entidades mantenedoras dos hospitais filantrópicos, sua condição de filantropia e as isenções fiscais usufruídas. Sobre o hospital foram considerados aspectos pertinentes ao perfil assistencial (de estrutura e de produção) e gerencial destes hospitais. No que tange à operadora, levantaram-se informações referentes à rede assistencial e à sua clientela 
(beneficiários), e características do seu dirigente, dos instrumentos de gestão e planejamento em geral, da gestão orçamentária e financeira, e dos recursos humanos e tecnologia de informação existentes. Em relação aos produtos comercializados, contemplaram-se os seguintes aspectos: número, tipo, abrangência, rede prestadora, segmentação, acomodações, exclusões, carências e coberturas, avaliação atuarial e mecanismos de regulação da rede. Foram ainda consideradas informações concernentes somente aos planos novo e antigo com maior número de beneficiários, relativas à caracterização dos beneficiários e ao volume de produção.

O questionário contemplou ainda, na forma de perguntas abertas, a percepção dos dirigentes sobre estratégias do setor hospitalar filantrópico frente à legislação dos planos de saúde e sobre possíveis ajustes à regulamentação, tendo em vista a especificidade do setor filantrópico.

O estudo teve caráter descritivo, sendo os resultados analisados a partir da constituição de três grupos: (i) operadoras de hospitais individuais registradas na ANS, (ii) operadoras de hospitais individuais não registradas na ANS e (iii) operadoras de conglomerados hospitalares registradas na ANS. Não foi verificada nenhuma ocorrência de operadora de conglomerado hospitalar não registrada na ANS.

Para a classificação da complexidade assistencial e do desempenho gerencial dos hospitais foram utilizados, respectivamente, o sistema de classificação da complexidade assistencial e o sistema de classificação de desenvolvimento gerencial de hospitais 6,7 , desenvolvidos no escopo do Estudo dos Hospitais Filantrópicos no Brasil 8. Este último baseia-se em uma perspectiva multidimensional, considerando sete dimensões da estrutura de gestão: (i) direção e instrumentos de planejamento, (ii) econômico-financeira, (iii) recursos humanos, (iv) logística, (v) serviços técnicos, (vi) tecnologia de informações e (vii) qualidade da assistência. As análises multidimensionais de desempenho gerencial, bem como as dimensões gerenciais aqui consideradas, são amplamente validadas na literatura 9,10 .

Como medidas do quanto a operadora está estruturada como empresa independente da entidade filantrópica que a abriga, foram consideradas a existência de Cadastro Nacional de Pessoa Jurídica (CNPJ) específico, de responsável pela operação dos planos com dedicação de quarenta horas semanais, de funcionários com vínculo específico e de orçamento e plano de contas próprios.

De forma complementar, para a análise da autonomia das operadoras em relação à entidade filantrópica que a abriga, foi construído um sis- tema de classificação considerando a existência de responsável específico pela área de planos de saúde (operadora) e seu poder de decidir, sem necessariamente consultar o dirigente da entidade, sobre dez ações gerenciais: contratação e corte de serviços de prestadores, realização de investimentos, contratação e demissão de pessoal, definição de política salarial, realização de aplicações financeiras, obtenção de empréstimos de curto e longo prazos e aprovação do orçamento. Foram consideradas sem autonomia (nível 0) as operadoras sem dirigentes específicos e aquelas que, apesar de terem dirigentes específicos, não tinham autonomia para nenhuma das ações gerenciais contempladas. Adicionalmente, cinco outros níveis de autonomia deram conta da classificação de operadoras com dirigentes específicos, conforme o número de ações gerenciais selecionadas: nível 1 , até duas condições de autonomia; nível 2, três ou quatro condições de autonomia; nível 3, cinco ou seis condições de autonomia; nível 4, sete ou oito condições de autonomia; e nível 5, nove ou dez condições de autonomia.

Para considerar o desenvolvimento gerencial das operadoras foi construído um sistema de classificação incluindo múltiplas dimensões da estrutura de gestão 2 , seguindo o mesmo racional utilizado no sistema de classificação do desenvolvimento gerencial de hospitais 6,7,8,9,10. Nesse caso, foram também consideradas as dimensões direção e instrumentos de planejamento, econômico-financeira e de tecnologia de informações, e adicionadas as de gestão da rede de prestadores das operadoras e dos direitos e responsabilidades dos usuários. As Tabelas 1, 2, 3, 4 e 5 apresentam as variáveis marcadoras utilizadas. Com base na verificação da presença das características indicadas por tais variáveis, cada uma das dimensões foi enquadrada em um nível de desenvolvimento - avançado, em desenvolvimento ou incipiente. Primeiramente observou-se se todos os requisitos exigidos no nível avançado foram atendidos. Não tendo sido, verificou-se a presença dos requisitos mínimos para o nível em desenvolvimento. Caso estes também não estivessem presentes, o grau de desenvolvimento da dimensão considerada foi classificado como incipiente. Em seguida atribuiu-se um escore à dimensão, tendo em vista o enquadramento descrito e os pontos correspondentes à contribuição proporcional dos requisitos adicionais presentes, pertinentes ao nível gerencial imediatamente superior. Os níveis incipiente e em desenvolvimento correspondem a escores que variam entre 0 e 4,9 pontos, e 5,0 e 9,9 pontos, respectivamente. O nível avançado corresponde a 10 pontos. O escore final de cada operadora foi definido como a média ponderada 
Estrutura da direção e instrumentos de planejamento.

\begin{tabular}{|c|c|c|c|}
\hline Condição & Incipiente & Em desenvolvimento & Avançado \\
\hline 1. Dirigente específico da operadora - existência & - & $x$ & $x$ \\
\hline \multicolumn{4}{|l|}{ 2. Dirigente executivo da operadora - número de horas dedicadas } \\
\hline \multicolumn{4}{|l|}{ à função e exclusividade na função } \\
\hline Pelo menos 40 horas semanais e função exclusiva & - & - & $x$ \\
\hline Entre 20 e 39 horas e/ou compartilhamento com outras funções & - & $x$ & - \\
\hline \multicolumn{4}{|l|}{ 3. Dirigente executivo da operadora } \\
\hline Pós-graduação + graduação & - & - & $x$ \\
\hline Curso superior completo (graduação completa) & - & $x$ & - \\
\hline \multicolumn{4}{|l|}{ 4. Práticas de planejamento (elementos analíticos) } \\
\hline Até 3 elementos & - & $x$ & - \\
\hline Mais de 3 elementos & - & - & $x$ \\
\hline \multicolumn{4}{|l|}{ 5. Práticas de planejamento (elementos propositivos) * } \\
\hline Até 3 elementos & - & $x$ & - \\
\hline Mais de 3 elementos & - & - & $x$ \\
\hline \multicolumn{4}{|l|}{ 6. Realização de investimentos (acima de 20 mil) } \\
\hline Até 3 alternativas & - & $x$ & - \\
\hline 4 ou mais & - & - & $x$ \\
\hline 7. Orçamento anual & - & $x$ & $x$ \\
\hline \multirow{2}{*}{\multicolumn{4}{|c|}{$\begin{array}{l}\text { 8. Monitoramento de indicadores de desempenho global } \\
\text { da operadora/autogestora ** }\end{array}$}} \\
\hline & & & \\
\hline 1 a 4 indicadores & - & $x$ & - \\
\hline Mais de 4 indicadores & - & - & $x$ \\
\hline
\end{tabular}

* Práticas de planejamento: (1) associação/parcerias com outras organizações; (2) investimento tecnológico - aquisição/reposição de equipamentos; (3) obras e ou reformas; (4) desenvolvimento de recursos humanos - formação e/ou treinamento; (5) estratégias de financiamento e endividamento; (5a) operações financeiras e de créditos; (6) modernização gerencial, incluindo novos sistemas e/ou treinamento gerencial; (7) incorporação de tecnologia de informação; (8) abertura de novos negócios; (9) planejamento de atividades de marketing.

** Indicadores de avaliação/monitoramento da autogestora: (1) taxa de sinistralidade; (2) taxa de utilização de serviços assistenciais; (3) custo por evento/custo por procedimento; (4) custo médio do exposto/custo médio do beneficiário; (5) relação receita/despesa por usuário; (6) nível de endividamento; (7) liquidez; (8) taxa de despesa administrativa.

dos escores obtidos em cada dimensão, considerando os seguintes pesos: 1,75, para direção e instrumentos de planejamento e direitos e responsabilidades dos usuários; 2,75, para estrutura econômico-financeira e sustentabilidade; 1,25 , para tecnologia da informação; e, 2,50, para gestão da rede de prestadores.

\section{Resultados}

A seguir são apresentadas uma breve descrição dos hospitais filantrópicos estudados e uma caracterização mais detalhada das operadoras. Os resultados relacionados às operadoras referemse ao conjunto de 96 operadoras de hospitais individuais registradas na ANS, 15 operadoras de hospitais individuais não registradas na ANS e 10 operadoras de conglomerados hospitalares re- gistradas na ANS. Ao todo são 111 operadoras em hospitais individuais quando na amostra eram 112 hospitais individuais; a diferença é explicada pelo fato de uma mesma operadora ser responsável pela operação de planos em dois hospitais simultaneamente.

\section{Localização geográfica, caracterização assistencial e gerencial dos hospitais filantrópicos com operadoras}

Os hospitais filantrópicos com operadoras de planos de saúde são, na maioria, de médio porte (51 a 150 leitos) e estão situados, predominantemente, em municípios do interior, de porte populacional médio.

Entre os hospitais individuais com operadoras registradas na ANS, predominam os de alta complexidade - realizam algum procedimento 


\begin{tabular}{|c|c|c|c|}
\hline Condição & Incipiente & Em desenvolvimento & Avançado \\
\hline \multicolumn{4}{|l|}{ 1. Função de responsabilidade na área econômico-financeira definida } \\
\hline (não necessariamente exclusiva) & - & $\mathrm{x}$ & $x$ \\
\hline 2. Nível superior de formação do responsável pela área econômico-financeira & - & $\mathrm{X}$ & $\mathrm{X}$ \\
\hline 3. Balanço fechado * & - & - & $x$ \\
\hline 4. Auditoria contábil independente & - & - & $x$ \\
\hline 5. Balancete periódico de patrimônio e resultados & - & - & $\mathrm{x}$ \\
\hline 6. Regras de depreciação patrimonial & - & - & $x$ \\
\hline 7. Prática de resseguro - "stop loss" & - & - & $x$ \\
\hline \multicolumn{4}{|l|}{ 8. Plano próprio de contas } \\
\hline Não ajustado a normas da ANS & - & $x$ & - \\
\hline Ajustado a normas da ANS & - & - & $X$ \\
\hline \multicolumn{4}{|l|}{ 9. Instrumentos de organização interna } \\
\hline $3-5$ & - & $x$ & - \\
\hline 6 ou mais & - & - & $x$ \\
\hline 10. Prática de análise atuarial & - & $x$ & $x$ \\
\hline 11. Práticas de avaliação de risco de beneficiários & - & - & $x$ \\
\hline 12. Utilização de índice de inadimplência & - & $x$ & $x$ \\
\hline 13. Reservas financeiras & - & $x$ & $x$ \\
\hline
\end{tabular}

ANS: Agência Nacional de Saúde Suplementar.

* Para respostas obtidas até 31 de março, foi considerado o balanço do ano anterior ao anterior; para respostas obtidas a partir de $1^{\circ}$ de abril, foi considerado o balanço do ano anterior.

de internação de alta complexidade e possuem leitos de cuidados intensivos (UTI) -, situados em municípios de maior porte populacional. Já entre os hospitais individuais com operadoras não registradas na ANS e conglomerados com operadoras registradas na ANS predominam unidades de baixa complexidade - clínicas básicas sem UTI. Entre os hospitais individuais com operadoras não registradas na ANS, destaca-se ainda que mais da metade constitui-se no único hospital em seus municípios.

De modo geral, observa-se que embora os hospitais filantrópicos com operadoras de planos de saúde prestem serviços para sua própria operadora e para outras do mercado, direcionam sua produção preponderantemente para o SUS (consultas, internações, atendimentos em hospital dia, atendimentos domiciliares e exames diagnósticos).

O desenvolvimento gerencial observado nos hospitais aqui estudados é ligeiramente superior ao do conjunto dos hospitais filantrópicos como um todo 8,11, destacando-se ainda a pouca diferenciação entre os três conjuntos contemplados. Os escores globais médios dos hospitais individuais com operadoras registradas na ANS, dos hospitais individuais com operadoras não regis- tradas na ANS e dos conglomerados com operadoras registradas na ANS foram, respectivamente, 5,6, 4,5 e 5,4. Note-se, ainda, que nos hospitais dos três conjuntos a dimensão melhor pontuada foi tecnologia de informação, enquanto a de menor pontuação foi qualidade assistencial.

\section{Localização geográfica e caracterização da autonomia e do desenvolvimento gerencial das operadoras dos hospitais filantrópicos}

No que tange à atividade de operação de planos de saúde, ela se concentra, para os três conjuntos de hospitais, nas regiões Sudeste e Sul e, predominantemente, fora das regiões metropolitanas. De maneira geral a própria operadora é a principal ou está entre as principais operadoras para as quais o hospital atua como prestador.

Considerando a independência das operadoras em relação à entidade a qual está vinculada, observa-se em geral que ela é pouco presente. Maior independência é encontrada nas operadoras de conglomerados hospitalares registradas na ANS, ainda que somente em duas das condições consideradas: $90 \%$ delas possuem plano de contas próprio e $70 \%$ registram responsável com dedicação de quarenta 
Estrutura em tecnologia de informações.

\begin{tabular}{|c|c|c|c|}
\hline Condição & Incipiente & Em desenvolvimento & Avançado \\
\hline \multicolumn{4}{|l|}{ 1. Acesso à Internet } \\
\hline Permanente (banda larga) & - & - & $x$ \\
\hline Discado & - & $x$ & - \\
\hline 2. Possui Intranet & - & - & $x$ \\
\hline \multicolumn{4}{|l|}{ 3. Sistemas de informações administrativas } \\
\hline 3 a 5 programas automatizados & - & $x$ & - \\
\hline 6 ou mais programas automatizados & - & - & $x$ \\
\hline \multicolumn{4}{|l|}{ 4. Sistemas de informações sobre usuários } \\
\hline Ao menos 5 indicadores/relatórios automatizados & - & $x$ & - \\
\hline 6 ou mais indicadores/relatórios automatizados & - & - & $\mathrm{x}$ \\
\hline \multicolumn{4}{|l|}{ 5. Pratica trocas eletrônicas (através da Internet) } \\
\hline \multicolumn{4}{|l|}{ com ANS e prestadores * } \\
\hline Ao menos 1 sistema & - & $x$ & - \\
\hline 3 ou mais sistemas & - & - & $x$ \\
\hline
\end{tabular}

ANS: Agência Nacional de Saúde Suplementar.

* Sistemas: recebimento de faturas; preenchimento do Sistema de Informação de Produtos; envio de cadastros de beneficiários da ANS.

Tabela 4

Estrutura de gestão da rede de prestadores das operadoras.

\begin{tabular}{|c|c|c|c|}
\hline Condição & Incipiente & Em desenvolvimento & Avançado \\
\hline \multicolumn{4}{|c|}{ 1. Cadastros de prestadores contratados contendo } \\
\hline \multicolumn{4}{|c|}{ documentações legais } \\
\hline Para alguns prestadores & - & $x$ & - \\
\hline Para todos os prestadores & - & - & $x$ \\
\hline \multicolumn{4}{|c|}{ 2. Realização de inspeção junto aos prestadores } \\
\hline 1 a 2 áreas de inspeção & - & $x$ & - \\
\hline 3 ou mais áreas de inspeção & - & - & $x$ \\
\hline \multicolumn{4}{|l|}{ 3. Existência de auditoria } \\
\hline Analítica e operacional & - & - & $x$ \\
\hline Apenas analítica ou operacional & - & $x$ & - \\
\hline 4. Prática de reciprocidade & - & - & $x$ \\
\hline \multicolumn{4}{|c|}{ 5. Formas de regulação/controles de utilização * } \\
\hline 2 a 3 condições & - & $\mathrm{x}$ & - \\
\hline 4 ou mais & - & - & $x$ \\
\hline
\end{tabular}

* Foram consideradas as seguintes: médico de família; médico de referência para pacientes especiais; porta de entrada; direcionamento ou referenciamento; autorização prévia; protocolos clínicos.

horas. Dessas operadoras, $50 \%$ referem ter predominância de funcionários próprios. Resultados intermediários são encontrados para as operadoras de hospitais individuais registradas na ANS, onde a única condição que se destaca positivamente é a existência de plano de conta próprio em 84,4\% delas; as demais condições são encontradas em menos da metade das operadoras estudadas. As operadoras de hospitais individuais não registradas na ANS, para todas as condições consideradas, apresentam resultados incipientes (Tabela 6). 
Estrutura de gestão dos direitos e responsabilidades dos usuários.

\begin{tabular}{|c|c|c|c|}
\hline Condição & Incipiente & Em desenvolvimento & Avançado \\
\hline 1. Existência de ouvidoria & - & - & $\mathrm{X}$ \\
\hline \multicolumn{4}{|c|}{$\begin{array}{l}\text { 2. Oferta de serviços pela Internet - informações sobre } \\
\text { prestadores da rede, sobre cuidados com a saúde, }\end{array}$} \\
\hline extratos de pagamentos/utilização & - & - & $x$ \\
\hline \multicolumn{4}{|c|}{ 3. Atendimento ao usuário - telefone 0800 ou } \\
\hline atendimento telefônico & - & $x$ & $\mathrm{x}$ \\
\hline \multicolumn{4}{|c|}{ 4. Realização de pesquisa de satisfação com usuários } \\
\hline Sistemática & - & - & $\mathrm{x}$ \\
\hline Eventual ou já realizou & - & $\mathrm{X}$ & - \\
\hline
\end{tabular}

Tabela 6

Independência das operadoras em relação às entidades e/ou hospitais filantrópicos. Brasil, 2003.

\begin{tabular}{|c|c|c|c|c|c|c|}
\hline \multirow[t]{2}{*}{ Condição } & \multicolumn{2}{|c|}{$\begin{array}{l}\text { Operadoras de hospitais } \\
\text { individuais registradas na } \\
\text { ANS }(n=96)\end{array}$} & \multicolumn{2}{|c|}{$\begin{array}{c}\text { Operadoras de hospitais } \\
\text { individuais não registradas } \\
\text { na ANS }(n=15)\end{array}$} & \multicolumn{2}{|c|}{$\begin{array}{l}\text { Operadoras de } \\
\text { conglomerados hospitalares } \\
\text { registradas na ANS ( } n=10 \text { ) }\end{array}$} \\
\hline & f & $\%$ & f & $\%$ & f & $\%$ \\
\hline CNPJ específico & 12 & 12,5 & 2 & 13,3 & 1 & 10,0 \\
\hline Responsável com dedicação de 40 horas & 45 & 46,9 & 5 & 33,3 & 7 & 70,0 \\
\hline Predominância de funcionários próprios & 18 & 18,8 & 0 & 0,0 & 5 & 50,0 \\
\hline Orçamento próprio & 19 & 19,8 & 1 & 6,7 & 3 & 30,0 \\
\hline Plano de contas próprio & 81 & 84,4 & 1 & 6,7 & 9 & 90,0 \\
\hline
\end{tabular}

ANS: Agência Nacional de Saúde Suplementar; CNPJ: Cadastro Nacional de Pessoa Jurídica.

Analisando-se o nível de autonomia a partir do sistema de classificação proposto, observa-se que $25 \%$ das operadoras de hospitais individuais registradas na ANS apresentam nível 0 de autonomia, ou seja, não têm responsável específico ou têm responsável específico, mas sem autonomia decisória em relação às dez ações gerenciais contempladas. As demais (75\%) distribuem-se nos outros níveis, sublinhando-se que somente 33\% se enquadram nos níveis 4 ou 5 , possuindo, de fato, maior autonomia em relação às entidades que as abrigam (Tabela 7). As ações gerenciais para as quais os dirigentes das operadoras mais freqüentemente têm autonomia são contratação de serviços (65,3\%), corte de serviços e demissão de pessoal $(62,1 \%)$ e contratação de pessoal $(61,1 \%)$.

A maior parte das operadoras de hospitais individuais não registradas na ANS (60\%) não tem nenhuma autonomia, e apenas uma destas operadoras enquadra-se no nível 5 (Tabela 7). As ações para as quais dirigentes específicos nesse grupo tiveram mais freqüentemente autonomia foram contratação de serviços e demissão de pessoal, ambas em $46,7 \%$ das operadoras.

Quanto às operadoras de conglomerados registradas na ANS, destaca-se a presença marcante de alguma autonomia (80\%), apesar de somente duas das dez situarem-se no nível 4 ou 5 (Tabela 7). As ações gerenciais para as quais os dirigentes dessas operadoras mais tiveram autonomia foram contratação e corte de serviços (50\% das operadoras).

Em relação ao nível de desenvolvimento gerencial das operadoras, observam-se escores globais consideravelmente baixos, inferiores a 5 , em todos os grupos de operadoras. As operadoras de hospitais individuais registradas na ANS tiveram como média ponderada 4,2; as não registradas na ANS obtiveram 1,4; e, finalmente, as de conglomerados registradas na ANS alcançaram 4,6 (Tabela 8). Apesar das baixas médias registradas, quatro operadoras de hospitais in- 
Tabela 7

Níveis de autonomia das operadoras de planos de saúde de hospitais filantrópicos. Brasil, 2003.

\begin{tabular}{|c|c|c|c|c|c|c|}
\hline \multirow[t]{2}{*}{ Nível de autonomia } & \multicolumn{2}{|c|}{$\begin{array}{l}\text { Operadoras de hospitais } \\
\text { individuais registradas na ANS } \\
\qquad(n=96)\end{array}$} & \multicolumn{2}{|c|}{$\begin{array}{l}\text { Operadoras de hospitais individuais } \\
\text { não registradas na ANS } \\
(n=15)\end{array}$} & \multicolumn{2}{|c|}{$\begin{array}{l}\text { Operadoras de conglomerados } \\
\text { hospitalares registradas na ANS } \\
\qquad(n=10)\end{array}$} \\
\hline & $f$ & $\%$ & $f$ & $\%$ & $f$ & $\%$ \\
\hline 0 & 24 & 25,0 & 9 & 60,0 & 2 & 20,0 \\
\hline 1 & 13 & 13,5 & 3 & 20,0 & 4 & 40,0 \\
\hline 2 & 12 & 12,5 & 1 & 66,7 & 2 & 20,0 \\
\hline 3 & 15 & 15,6 & 1 & 66,7 & - & - \\
\hline 4 & 11 & 11,5 & - & - & 1 & 10,0 \\
\hline 5 & 21 & 21,9 & 1 & 66,7 & 1 & 10,0 \\
\hline
\end{tabular}

ANS: Agência Nacional de Saúde Suplementar.

dividuais e uma de conglomerado registradas na ANS destacaram-se com escore entre 7,0 e 8,9 pontos.

No que concerne às operadoras de hospitais individuais e de conglomerados registradas na ANS, a dimensão melhor pontuada foi tecnologia de informação, que obteve os escores, respectivamente, de 6,6 e 6,4. Nas operadoras de hospitais individuais não registradas na ANS, a dimensão melhor pontuada foi direitos e responsabilidades dos usuários, com 2,6 pontos (Tabela 8). A dimensão com menor pontuação em operadoras de hospitais individuais e de conglomerados registradas na ANS foi a econômico-financeira, com 3,3 e 3,4 pontos, respectivamente. As operadoras de hospitais individuais não registradas na ANS, embora tenham obtido somente 1,2 ponto nessa dimensão, mostraram pior desempenho na gestão da rede prestadora (1,0 ponto), dimensão na qual as operadoras de hospitais individuais e de conglomerados registradas na ANS obtiveram médias de 4,5 e 4,2.

Quanto à percepção dos dirigentes sobre as principais repercussões da regulamentação sobre as operadoras filantrópicas, os temas que mais se destacaram foram: reajuste de preços, realização de maiores investimentos em tecnologias gerenciais (sistemas de informações, sistemas de planejamento e outros), aumento dos custos administrativos advindo do ajuste organizacional necessário à alimentação dos sistemas introduzidos pela ANS (fundamentalmente pelos de informação contábil-financeira e dos produtos) e capacitação de dirigentes e técnicos para fazer frente às exigências da nova legislação.

No que se refere aos produtos comercializados, as operadoras não registradas na ANS apresentam importante diferenciação, compre- endendo de planos com características similares aos registrados, até simples práticas de parcelamentos do pagamento referente a procedimentos específicos, incluindo os chamados cartões descontos. Embora nesse conjunto tenham sido estudadas apenas 15 operadoras, supõe-se que a condição de produtos não registrados possa ser mais diversa e numerosa do que a verificada, dado que foi neste grupo que houve maior número de recusas em participar.

Nas operadoras de hospitais individuais e de conglomerados registradas na ANS, destaca-se o número de produtos e beneficiários vinculados a planos individuais ou familiares, superior a $50 \%$ do total, diferentemente dos demais segmentos de operadoras do mercado que operam com mais de $75 \%$ de beneficiários vinculados a produtos coletivos.

Sublinha-se ainda o elevado percentual de clientela idosa encontrado nos três grupos de operadoras, provavelmente pela limitada renovação das carteiras, com ingressos na base da pirâmide, particularmente nos planos individuais. A concentração de riscos nessa faixa etária é sabidamente superior, sempre com alguma conseqüência para o grau de solvência do plano. Como esperado, a concentração de idosos é maior nos planos individuais, uma vez que planos coletivos tendem a operar, mais freqüentemente, para a população economicamente ativa.

Nas operadoras de hospitais individuais registradas na ANS verificou-se uma utilização maior de rede contratada, em contraposição ao observado nas de conglomerados registradas na ANS, provavelmente mais refletindo a localização predominante dos primeiros em municípios de maior porte e, portanto, abrigando maior competitividade, do que uma maior capacidade 
Médias ponderadas das dimensões gerenciais nas operadoras de planos de saúde do segmento filantrópico. Brasil, 2003.

\begin{tabular}{|c|c|c|c|}
\hline Dimensões & $\begin{array}{l}\text { Operadoras de hospitais } \\
\text { individuais registradas na } \\
\text { ANS }(n=96)\end{array}$ & $\begin{array}{l}\text { Operadoras de hospitais } \\
\text { individuais não registradas } \\
\text { na ANS }(n=15)\end{array}$ & $\begin{array}{l}\text { Operadoras de } \\
\text { conglomerados hospitalares } \\
\text { registradas na ANS }(n=10)\end{array}$ \\
\hline Direção e instrumentos de planejamento & 3,4 & 1,4 & 4,5 \\
\hline Estrutura econômico-financeira & 3,3 & 1,2 & 3,4 \\
\hline Tecnologia da informação & 6,6 & 1,3 & 6,4 \\
\hline Gestão da rede prestadora & 4,5 & 1,0 & 4,2 \\
\hline Direitos e responsabilidades dos usuários & 4,4 & 2,6 & 5,7 \\
\hline Escore global & 4,2 & 1,9 & 4,6 \\
\hline
\end{tabular}

ANS: Agência Nacional de Saúde Suplementar

assistencial no conjunto dos hospitais componentes dos conglomerados.

Nas operadoras de hospitais individuais, registradas e não registradas na ANS, a abrangência dos produtos é majoritariamente municipal e de grupos de municípios, em alguns casos, o que é condizente com a base local destas operadoras. As de conglomerados registradas na ANS, por sua vez, tendem a oferecer mais planos com abrangência de grupos de municípios, dado que há vários conglomerados com hospitais em mais de um município.

Em função das exigências de cobertura de rol mínimo de procedimentos, para os planos novos há prática de contratação de terceiros. Diferentemente, nos planos antigos, as coberturas contratadas estão relacionadas aos procedimentos disponíveis nos respectivos hospitais, prevalecendo uma elevada lista de exclusões, o que confirma as limitações de boa parte dos hospitais em suprir por si as exigências legais dos planos novos. Há, em todos os grupos, praticamente ausência de planos odontológicos.

Em termos de oferta de programas mais estruturados de atenção, basicamente de prevenção para riscos determinados (diabetes, hipertensão, outros), há importante carência, confirmando que o modelo de atenção predominante é ainda curativo. Não há operadoras que ofertem programas no campo da saúde do trabalhador.

É limitada a emissão das notas técnicas atuariais para os planos antigos em todos os grupos de operadoras. Há também planos novos em que não se realizam análises atuariais.

Finalmente, em relação aos controles de utilização aplicados aos diversos produtos, prevalecem, em todos os grupos de operadoras, formas clássicas, tais como a co-participação nos eventos e a emissão de senhas para atendimento. Lógicas mais avançadas são bastante limitadas, como prática de segunda opinião médica ou utilização de protocolos assistenciais.

\section{Discussão}

As operadoras de hospitais filantrópicos se situam em condições especiais no mercado: de um lado, aquelas que são únicas no município, concorrendo na prática com o próprio SUS, diferenciando seus produtos fundamentalmente em termos de hotelaria; do outro, aquelas localizadas em municípios de maior porte, que têm como fator competitivo os seus preços, inferiores aos do mercado, propiciados pelos incentivos fiscais disponíveis, pelo fato de o prestador ser próprio e pela amortização de investimentos decorrente da oferta concomitante de serviços ao SUS.

Outra característica do segmento é sua grande interiorização. A imensa maioria encontra-se em pequenos municípios, operando planos de abrangência municipal e com um número médio de beneficiários pequeno. Essas características praticamente configuram um mercado particular, com limitações quanto à expansão e riscos referentes à estabilidade e solvência do negócio no médio e longo prazos. A necessária oferta do rol de procedimentos para os planos novos e as obrigações/induções para migração dos planos antigos são claros constrangimentos para as operadoras de hospitais filantrópicos, que padecem de escala quanto ao número médio de beneficiários e, portanto, têm limitações quanto ao compartilhamento do risco entre os segurados. Por outro lado, no curto prazo, a arrecadação de recursos advindos dos planos é uma potencial fonte de receitas da entidade, expectativa que para se confirmar exige estudos mais específicos e detalhados acerca dos dados econômico-financeiros. Além disso, há relato na literatura apontando a inte- 
riorização, nos Estados Unidos, como condição facilitadora para o sucesso da operação de planos próprios em sistemas integrados de serviços de saúde, tendo em vista que a localização em áreas rurais acarreta uma competição limitada, altas taxas de utilização de serviços, maiores margens de lucro em função do baixo preço e maior aceitação dos prêmios a serem pagos por parte dos compradores de planos (empregadores) 12 .

Diferentemente do resto do mercado da saúde suplementar, observa-se um peso maior dos planos individuais em relação aos produtos coletivos, o que também afeta o compartilhamento do risco entre a população segurada. No segmento filantrópico, a maior freqüência de planos individuais ou familiares parece ser decorrente tanto de aspectos históricos (os antigos associados) quanto do mercado onde operam, com menor dinâmica econômica e, portanto, reduzida demanda empresarial por planos coletivos. No entanto, este predomínio de planos individuais também pode ser decorrente da limitada capacidade empreendedora das filantrópicas, uma vez que o mercado de planos coletivos seria mais disputado e melhor conquistado por operadoras mais profissionalizadas e mais atuantes no mercado.

Outro elemento que acaba por condicionar o futuro desse segmento é a sua própria condição de gestão. O estudo apresenta uma importante debilidade das operadoras filantrópicas em termos de condições gerenciais, demonstrada por meio dos níveis de desenvolvimento gerencial das suas operadoras. Ainda que esse segmento de entidades e "operadoras" estudadas sejam positivamente diferenciadas em relação ao conjunto total de hospitais filantrópicos 8,11 , não há dúvidas quanto às fragilidades encontradas. Boas condições gerenciais seriam fundamentais para o setor reagir no mercado e buscar melhor desempenho tanto no equilíbrio interno quanto na competição com outros segmentos que disputam o mesmo espaço, particularmente as cooperativas médicas do interior do país. Aqui reside outro ponto a merecer atenção, tanto dos próprios dirigentes do segmento quanto do órgão regulador, no momento da definição de estratégias internas ou externas indutivas ao desenvolvimento do segmento.

Como causa e conseqüência do incipiente desenvolvimento gerencial, as operadoras filantrópicas não se constituem exatamente em empresas estruturadas de forma independente da entidade mantenedora. Mesmo as operadoras registradas na ANS, seja de hospitais individuais, seja de conglomerados hospitalares, apresentam-se muito imbricadas com suas entidades e com pouca autonomia para tomar decisões que, em última instância, podem ter impactos no seu desempenho gerencial.

Tais características parecem influenciar sua operação: não operam exatamente dentro do mercado de planos, onde se encontra a maior parte das operadoras - seguradoras, empresas de medicina de grupo e cooperativas médicas. São planos que, na sua imensa maioria, não funcionam a partir de operadoras típicas, mas, sim, a partir de "dentro da própria entidade ou hospital”, quase sempre em condições limitadas de estruturas de gestão e com pouca autonomia. Igualmente, é natural que as poucas operadoras, quando constituídas como tais, se mantenham dentro das entidades filantrópicas que as constituíram, sob pena de não gozar os benefícios da filantropia, sempre determinados pela condição geral da entidade.

Quando os hospitais ainda são prestadores para outras operadoras (na maior parte dos casos), tende a configurar-se uma condição positivamente diferenciada, uma vez que os preços praticados para outras operadoras tendem a ser mais altos que os do SUS. Os dados disponíveis não permitiram tais análises e conclusões mais seguras, pois os balanços não possuíam, em geral, homogeneidade na sua apresentação. Entretanto, provavelmente as margens de lucro dos hospitais são superiores quando estes prestam serviços a outras operadoras.

$\mathrm{O}$ estudo evidencia claramente que, tendo em vista a fragilidade gerencial dessas instituições e, ainda, a precariedade dos seus resultados econômico-financeiros, estas instituições que, no seu formato, poderiam ser comparadas às Health Maintenance Organizations (HMOs) americanas, delas se distanciam no lugar que ocupam no mercado e nas estratégias adotadas: enquanto as operadoras oriundas de entidades filantrópicas, no Brasil, se mantêm pulverizadas e com uma clientela basicamente local, as HMOs estão, desde o início dos anos noventa, em um processo de fusão, em busca de economias de escala com estratégias ativas de procura de maiores parcelas de mercado ${ }^{13}$.

No que concerne à qualidade assistencial, este estudo aponta para resultados que contrastam com evidências encontradas nos Estados Unidos, onde se verifica que os planos de saúde comercializados por instituições sem finalidade de lucro obtiveram melhor escore do que os de operadoras com finalidade de lucro nos seis principais indicadores de qualidade do sistema Health Plan Employer Data and Information Set (HEDIS) 14.

Em suma, este estudo evidencia, fundamentalmente, a heterogeneidade presente na atividade de operação de planos de saúde do setor 
filantrópico hospitalar e dos produtos por ele comercializados. Aponta, ainda, que essa atividade resulta fundamentalmente de uma estratégia de sobrevivência econômica dessas entidades hospitalares, que prestam ao SUS, na sua maioria, serviços de média complexidade, pior remunerados pela tabela do Sistema de Informações Hospitalares do SUS (SIH/SUS) do que os de alta complexidade. Ademais, é notória a interiorização desse segmento hospitalar, o que faz com que seus planos de saúde sejam de abrangência municipal e os serviços por ele oferecidos estejam profundamente imbricados com a produção efetuada para o SUS. Enfim, este estudo remete para a necessidade de maior aprofundamento sobre o papel da operação de planos de saúde pelo setor filantrópico hospitalar, que, atuando ou não na saúde suplementar, possui importância estratégica no SUS, especialmente no interior do país.

\section{Resumo}

Este artigo caracteriza os hospitais filantrópicos com operadoras de planos de saúde, as operadoras em si, considerando seu nível de autonomia em relação aos hospitais e seu grau de desenvolvimento gerencial a partir de um estudo nacional. Foi constituída uma amostra aleatória de hospitais individuais e considerado o universo dos conglomerados hospitalares. Considerando as recusas e substituições restaram 112 hospitais individuais e dez conglomerados hospitalares. As operadoras de hospitais filantrópicos não operam exatamente dentro do mercado de planos, onde se encontra a maior parte das operadoras - seguradoras, empresas de medicina de grupo e cooperativas médicas. Não se constituem como operadoras típicas, mas funcionam a partir de "dentro da própria entidade ou hospital", quase sempre em condições limitadas de estruturas de gestão e com pouca autonomia em relação às entidades que as abrigam. Observa-se um peso maior dos planos individuais em relação aos produtos coletivos, diferentemente do resto do mercado, o que além de outras determinações pode também ser decorrente de sua limitada capacidade gerencial.

Planos de Saúde; Hospitais Filantrópicos; Assistência Hospitalar

\section{Colaboradores}

$\mathrm{O}$ artigo resulta de pesquisa desenvolvida por todos os autores, sob a coordenação de M. A. D. Ugá. Todos os autores participaram da concepção e estruturação, bem como da análise dos dados e redação do texto.

\section{Agradecimentos}

Este trabalho sintetiza os resultados da pesquisa de âmbito nacional, Dimensionamento dos Planos de Saúde Comercializados por Hospitais Filantrópicos, desenvolvida ao longo de 2003 e 2004, pela Escola Nacional de Saúde Pública Sergio Arouca da Fundação Oswaldo Cruz, resultante de concorrência pública promovida pela Agência Nacional de Saúde Suplementar (ANS) do Ministério da Saúde. Esta pesquisa foi regida pela carta acordo 47-478/03, firmada entre a Fundação para o Desenvolvimento Científico e Tecnológico em Saúde e a ANS. 


\section{Referências}

1. Agência Nacional de Saúde Suplementar, Ministério da Saúde. Caderno de informação da saúde suplementar: beneficiários, operadoras e planos. http://www.ans.gov.br/portal/upload/informaco esss/caderno_informaca_03_2006.pdf (acessado em 13/Jun/2007).

2. Ugá MAD, Portela MC, Vasconcellos MM, Barbosa PR, Lemos SML, Gerschman S. Dimensionamento dos planos de saúde comercializados por hospitais filantrópicos. http://www.ans.gov.br/portal/site/ Biblioteca/biblioteca_topico_17702.asp (acessado em 13/Jun/2007).

3. Brasil. Lei no. 9.656, de 03 de junho de 1998. Dispõe sobre os planos e seguros privados de assistência à saúde. Diário Oficial da República Federativa do Brasil 1998; 4 jun.

4. Brasil. Medida Provisória no ${ }^{\circ}$. 2.177-44, de 24 de agosto de 2001. Dispõe sobre os planos e seguros privados de assistência à saúde, regulamentando a Lei no. 9.656, de 03 de junho de 1998. Diário Oficial da República Federativa do Brasil 2001; 27 ago.

5. Brasil. Lei $\mathrm{n}^{\circ}$. 9.661, de 28 de janeiro de 2000. Cria a Agência Nacional de Saúde Suplementar (ANS) e dá outras providências. Diário Oficial da República Federativa do Brasil 2000; 29 jan.

6. Portela MC, Lima, SML, Barbosa PR, Vasconcellos MM, Ugá MAD, Gerschman S. Caracterização assistencial dos hospitais filantrópicos no Brasil. Rev Saúde Pública 2004; 38:811-8.
7. Lima SML, Barbosa PR, Portela MC, Ugá MAD, Vasconcellos MM, Gerschamn S. Caracterização gerencial dos hospitais filantrópicos no Brasil. Cad Saúde Pública 2004; 20:1249-61.

8. Barbosa PR, Portela MC, Ugá MAD, Vasconcellos MM, Lima SML, Gerschman S. Hospitais filantrópicos no Brasil. Rio de Janeiro: Banco Nacional de Desenvolvimento Econômico e Social; 2002. (BNDES Social, 5).

9. Griffith JR. Championship management for healthcare organizations. J Healthc Manag 2000; 45:17-31

10. Griffith JR, Alexander JA, Jelinek RC. Measuring comparative hospital performance. J Healthc Manag 2002; 47:41-57.

11. Lima SML, Portela MC, Ugá MAD, Barbosa PR, Gerschman S, Vasconcellos MM. Hospitais filantrópicos e a operação de planos de saúde próprios no Brasil. Rev Saúde Pública 2007; 41:116-23.

12. Burns LR, Thorpe DP. Why provider-sponsored health plans don't work. Healthc Financ Manage 2001; Suppl:12-6.

13. Stats \& facts. Do hospitals benefit from owning HMOs? Manag Care Interface 2000; 13:44-5.

14. HEDIS: looking at the numbers. Clinically, for-profits lag not-for-profits. Manag Care 1998; 7:42-3.

Recebido em 17/Jan/2006

Versão final reapresentada em 15/Jun/2007 Aprovado em 25/Jun/2007 